\title{
Liquid exfibration and optoelectronic devices of fibrous phosphorus
}

\author{
Guoqing Zhang, \\ Xianlin $\mathrm{Qu},{ }^{\dagger,}$ He Ma, ${ }^{\ddagger}$ Changzeng Fan, ${ }^{\mathrm{G}}$ and Yongzhe Zhang ${ }^{*,+\|}$ \\ †ey Laboratory of Advanced Functional Materials, Education Ministry of China, "Institute of Microstructure \\ and Property of Advanced Materials, "College of Materials Science and Engineering, and ${ }^{\#}$ College of Applied \\ Sciences, Beijing University of Technology, Beijing 100124, China \\ ${ }^{\S}$ Guangxi Key Laboratory of Optical and Electronic Materials and Devices, Guilin University of Technology, \\ Guilin 541004, China \\ ${ }^{\perp}$ School of Physics and Nuclear Energy Engineering, Beihang University, Beijing 100083, China \\ 'State Key Laboratory of Metastable Materials Science and Technology, Yanshan University, Qinhuangdao \\ 066004, China
}




\section{Experimental procedure}

Typical synthetic procedure: Silica glass ampoules were flushed with tap water containing detergents. Subsequently, these ampoules were cleaned sequentially by ultrasound with acetone, ethanol, and deionized water for 15 minutes each. Then these ampoules were placed in a vacuum drying box at $80^{\circ} \mathrm{C}$ under vacuum for 12 hours. $\mathrm{Sn}(40 \mathrm{mg}), \mathrm{SnI}_{4}(20 \mathrm{mg})$, and red phosphorus $(1 \mathrm{~g})$ were sealed in an evacuated silica glass ampoule. These ampoules were rapidly heated up to $650-550^{\circ} \mathrm{C}$ and then gradually cooled down to $550-450^{\circ} \mathrm{C}$. Then the products located within the walls of the colder end of glass ampoule. Unfortunately, black phosphorus is also produced under the same conditions. The FP was separated by visual examination from the black phosphorus.

XRD diffractogram: The XRD patterns for fibrous phosphorus were measured using a Bruker D8 diffractometer, using $\mathrm{Cu}$ Kal radiation at $\lambda=1.5406 \AA$. Samples were prepared by dispersing finely ground powders of the materials onto a silicon sample holder.

The Raman spectrum: It was recorded by a Witec alpha300 confocal Raman microscope using $532 \mathrm{~nm}$ laser excitation. Samples were transferred to $\mathrm{SiO}_{2}(300 \mathrm{~nm}) / \mathrm{Si}$ substrates.

The liquid exfibration experiment: FP bulks were exfibrated by alcoholic solution for $1 \mathrm{~h}$ without being sealed. The ultrasonic power was kept at $100 \mathrm{~W}$ (JY98-111DN cell disruptor). During the liquid exfibration experiment, the temperature of the solution was kept cool by ice to avoid ethanol volatilization. The liquid exfibration samples were drop-coated onto $\mathrm{SiO}_{2}$ $(300 \mathrm{~nm}) / \mathrm{Si}$ substrates and carbon micro grids. Then these samples were dried at $80^{\circ} \mathrm{C}$ for 12 hours to remove residual alcohol to prepare for TEM.

Transmission electron microscopy: Crystallites of fibrous phosphorus were examined using a FEI Tecnai G2 T20 at an acceleration voltage of $200 \mathrm{kV}$.

Fabrication of field effect transistors: To fabricate the field effect transistors, the $\mathrm{SiO}_{2}(300 \mathrm{~nm}) / \mathrm{Si}$ substrate were treated by oxygen plasma to increase hydrophilicity and remove residue of substrate. Then the liquid exfibrated samples were transferred to substrate by spin coating process, followed by vacuum drying at 80 -degree Celsius for $10 \mathrm{~h}$. The devices were patterned by UV lithography (SUSS MJB4) and two electrodes of $\mathrm{Ti} / \mathrm{Au}(10 \mathrm{~nm} / 100 \mathrm{~nm})$ were deposited by the e-beam evaporation system (HHV FL400).

Measurement of electronic and optoelectronic properties: The photoelectric properties of fibrous phosphorus were tested using a semiconductor device analyzer (Agilent B1500A) in ambient air. A supercontinuum light source (SuperK Extreme, NKT Photonics) with tunable light was used for illumination. We used a Nikon Ni-U microscope to focus on the light spot. Photoresponsivity as a function of time under modulated light intensity $(\lambda=532 \mathrm{~nm}$, laser intensity $=0.516$ $\left.\mathrm{mW} / \mathrm{cm}^{2}, \mathrm{~V}_{\mathrm{ds}}=2 \mathrm{~V}\right)$.

\section{Identity the direction of FP fibers:}

The SEM image of a single one (Figure S1b) shows that it has a smooth surface. The single-crystal structure of individual fibers was ascertained by transmission electron microscopy (TEM), as shown in Figure 2a. The selected area diffraction (SAED) pattern recorded along the fibers (inset) can be indexed as (100). In addition, FP is a layer-like structure, and the (001) plane is connected via van der Waals forces; i.e. another plane along the fibers is (001). This confirmed that the direction of the fiber's length is undeniably parallel to the (001) plane. Thus, the direction of the axis of the FP fibers is [010].

\section{Fitting the Schottky barrier:}

The flow of carriers is affected by the Schottky barrier because it inhibits thermal charge injection. The nonlinear characteristic of the $\mathrm{I}_{\mathrm{ds}}-\mathrm{V}_{\mathrm{ds}}$ curve for two Schottky junctions can be expressed by the thermoionic emission model ${ }^{29}$. The voltage $\left(\mathrm{V}_{\mathrm{ds}}\right)$ and current (Ids) are given by 


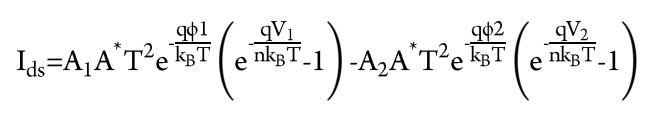

where $h$ is Planck' s constant, $\mathrm{A}^{*}=4 \pi q k_{\mathrm{B}}^{2} / h^{3}$ is the effective Richardson constant, $\mathrm{n}$ is the diode ideality factor, $k_{\mathrm{B}}$ is the Boltzmann constant, $\mathrm{q}$ is the electron charge, and $\mathrm{T}(300 \mathrm{~K})$ is the temperature. $\mathrm{A}_{1}, \mathrm{~A}_{2}$ are the contact areas between the sample and the electrodes. $V_{1}$, and $V_{2}$ are the voltage drops on the Schottky junctions. $q \phi_{1}$ and $q \phi_{2}$ are the heights of the Schottky barriers for diode 1 and diode 2, respectively.

\section{Optical micrograph and SEM image of exfibrated FP}

(a)

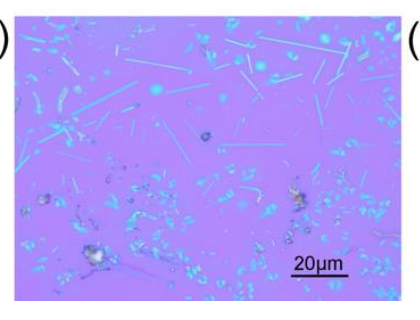

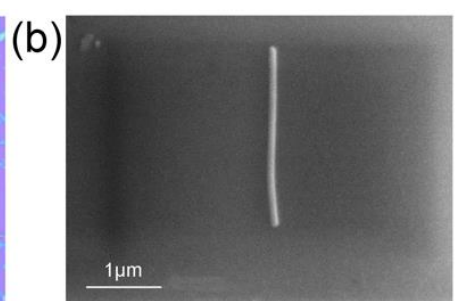

Figure S1. Characterization of liquid exfibrated fibrous phosphorus. a) Optical micrograph of liquid exfibrated fibrous phosphorus. b) SEM image of liquid exfibrated fibrous phosphorus. 